\title{
Chromatic Turán problems and a new upper bound for the Turán density of $\mathcal{K}_{4}^{-}$
}

\author{
John Talbot \\ Department of Mathematics, University College London, UK \\ E-mail:talbot@math.ucl.ac.uk
}

We consider a new type of extremal hypergraph problem: given an $r$-graph $\mathcal{F}$ and an integer $k \geq 2$ determine the maximum number of edges in an $\mathcal{F}$-free, $k$-colourable $r$-graph on $n$ vertices.

Our motivation for studying such problems is that it allows us to give a new upper bound for an old problem due to Turán. We show that a 3 -graph in which any four vertices span at most two edges has density less than $\frac{33}{100}$, improving previous bounds of $\frac{1}{3}$ due to de Caen [1], and $\frac{1}{3}-4.5305 \times 10^{-6}$ due to Mubayi [9].

Keywords: Extremal combinatorics, Turán-type problems, Hypergraphs

Given an $r$-graph $\mathcal{F}$ the Turán number $\operatorname{ex}(n, \mathcal{F})$ is the maximum number of edges in an $n$-vertex $r$-graph not containing a copy of $\mathcal{F}$. The Turán density of $\mathcal{F}$ is

$$
\pi(\mathcal{F})=\lim _{n \rightarrow \infty} \frac{\operatorname{ex}(n, \mathcal{F})}{\left(\begin{array}{l}
n \\
r
\end{array}\right)} .
$$

For 2-graphs the Turán density is determined completely by the chromatic number of $\mathcal{F}$, but for $r \geq 3$ there are very few $r$-graphs for which $\pi(\mathcal{F})$ is known. (Examples of 3-graphs for which $\pi(\mathcal{F})$ is now known include the Fano plane [2], $\mathcal{F}=\{a b c, a b d, a b e, c d e\}$ [8] and $\mathcal{F}=\{a b c, a b d, c d e\}$ [7].)

The two most well-known problems in this area are to determine $\pi\left(\mathcal{K}_{4}\right)$ and $\pi\left(\mathcal{K}_{4}^{-}\right)$, where $\mathcal{K}_{4}=$ $\{a b c, a b d, a c d, b c d\}$ is the complete 3 -graph on 4 vertices and $\mathcal{K}_{4}^{-}=\{a b c, a b d, a c d\}$ is the complete 3 -graph on 4 vertices with an edge removed. For $\pi\left(\mathcal{K}_{4}\right)$ we have the following bounds due to Turán and Chung and $\mathrm{Lu} \mathrm{[3]} \mathrm{respectively}$

$$
\frac{5}{9} \leq \pi\left(\mathcal{K}_{4}\right) \leq \frac{3+\sqrt{17}}{12}=0.59359 \ldots
$$

Although the problem of determining $\pi\left(\mathcal{K}_{4}\right)$ is an extremely natural question in some respects the problem of determining $\pi\left(\mathcal{K}_{4}^{-}\right)$is even more fundamental since $\mathcal{K}_{4}^{-}$is the smallest 3-graph satisfying $\pi(\mathcal{F}) \neq 0$. Note also that this problem may be restated as follows: determine the maximum density of a 3-graph in which any four vertices span at most two edges. 
The problem of determining $\pi\left(\mathcal{K}_{4}^{-}\right)$has been considered by many people, including Turán [12], Erdős and Sós [5], Frankl and Füredi [6], de Caen [1] and Mubayi [9]. Previously the best known bounds were

$$
\frac{2}{7} \leq \pi\left(\mathcal{K}_{4}^{-}\right) \leq \frac{1}{3}-\left(4.5305 \times 10^{-6}\right)
$$

The upper bound was proved by Mubayi [9], improving on the upper bound $\pi\left(\mathcal{K}_{4}^{-}\right) \leq 1 / 3$, due to de Caen [1]. The lower bound is from a construction due to Frankl and Füredi [6].

Our main result is the following theorem, improving the upper bound for $\pi\left(\mathcal{K}_{4}^{-}\right)$.

Theorem 1 The Turán density of $\mathcal{K}_{4}^{-}$satisfies

$$
\frac{2}{7} \leq \pi\left(\mathcal{K}_{4}^{-}\right)<\frac{33}{100}
$$

The proof of this result leads naturally to a new general class of extremal problems which we call chromatic Turán problems. These are questions of the form: given an $r$-graph $\mathcal{F}$ and an integer $k \geq 2$ determine the maximum number of edges in an $\mathcal{F}$-free, $k$-colourable $r$-graph on $n$ vertices. (Recall that an $r$-graph is $k$-colourable iff its vertices can be partitioned into $k$ classes, none of which contain an edge.) We denote this quantity by $\operatorname{ex}_{k}(n, \mathcal{F})$. The corresponding $k$-chromatic Turán density is then defined to be

$$
\pi_{k}(\mathcal{F})=\lim _{n \rightarrow \infty} \frac{\operatorname{ex}_{k}(n, \mathcal{F})}{\left(\begin{array}{l}
n \\
r
\end{array}\right)} .
$$

The following lemma shows that any upper bound for the chromatic Turán density $\pi_{3}\left(\mathcal{K}_{4}^{-}\right)$will yield an upper bound for the ordinary Turán density $\pi\left(\mathcal{K}_{4}^{-}\right)$. (The proof of Theorem 1 actually requires a slightly stronger result but the principle is the same.)

Lemma 1 If $\pi_{3}=\pi_{3}\left(\mathcal{K}_{4}^{-}\right)$and $\pi=\pi\left(\mathcal{K}_{4}^{-}\right)$then

$$
\pi \leq \frac{2+\pi_{3} / \pi}{9}
$$

To prove Lemma 1 we first note that if $\mathcal{F}$ is a $\mathcal{K}_{4}^{-}$-free 3 -graph with $n$ vertices and $m$ edges then it satisfies

$$
m n=\sum_{x y \in V^{(2)}} d_{x y}^{2}+q_{1},
$$

where $q_{1}$ is the number of 4 -sets in $\mathcal{F}$ spanning exactly one edge. Lemma 1 is proved by giving a lower bound for $q_{1}$.

This idea of giving a lower bound for $q_{1}$ was previously used by Mubayi [9] (he used supersaturation to achieve this). Our approach is quite different in that it involves a chromatic Turán problem.

To be more precise the key idea used in the proof of Lemma 1 is as follows. Let $\mathcal{F}$ be a $\mathcal{K}_{4}^{-}$-free 3 -graph of order $n$ and let $u v w \in \mathcal{F}$. If $E_{i j}=\{k: i j k \in \mathcal{F}\}$ then $E_{u v}, E_{u w}$ and $E_{v w}$ are pairwise disjoint. We say that an edge $x y z \in \mathcal{F}$ is bad relative to the edge $u v w$ if $x y z \cap u v w=\emptyset$ and none of the following 
4-sets span exactly one edge: $u v w x, u v w y, u v w z, x y z u, x y z v, x y z w$. It is straightforward to check that if $x y z$ is bad relative to $u v w$ then $x y z \subset E_{u v} \cup E_{u w} \cup E_{v w}$ and $x y z$ is not contained entirely in either $E_{u v}, E_{u w}$ or $E_{v w}$. Hence the collection of bad edges relative to $u v w$ is 3-colourable. This fact can then be used to give a lower bound for $q_{1}$ in terms of the 3-chromatic Turán density $\pi_{3}\left(\mathcal{K}_{4}^{-}\right)$.

One obvious reason why chromatic Turán problems do not seem to have been considered before is that for 2-graphs they are rather uninteresting. If $K_{t}$ is the complete 2-graph of order $t$ then Turán's theorem determines not only their Turán numbers but also their chromatic Turán numbers.

Theorem 2 (Turán[11]) If $t \geq 3$ then the unique $K_{t}$-free graph with $n$ vertices and ex $\left(n, K_{t}\right)$ edges is the complete $(t-1)$-partite graph whose vertex classes are as equal as possible in size.

Corollary 1 If $t \geq 3$ and $k \geq 2$ then

$$
\operatorname{ex}_{k}\left(n, K_{t}\right)= \begin{cases}\operatorname{ex}\left(n, K_{t}\right), & k \geq t-1 \\ \operatorname{ex}\left(n, K_{k+1}\right), & k \leq t-2 .\end{cases}
$$

For general $r$-graphs with $r \geq 3$ the problems of determining chromatic and ordinary Turán numbers seem to be genuinely different. While the extremal $K_{t}$-free 2 -graph is not only $K_{t}$-free but also $(t-1)$ colourable this does not seem to be the case in general. For example the conjectured extremal $\mathcal{K}_{4}^{-}$-free 3-graph has rather large chromatic number.

In order to make use of Lemma 1 we need an upper bound on $\pi_{3}\left(\mathcal{K}_{4}^{-}\right)$. To achieve this we first consider $\pi_{2}\left(\mathcal{K}_{4}^{-}\right)$.

Theorem 3 The 2-chromatic Turán density $\pi_{2}\left(\mathcal{K}_{4}^{-}\right)$satisfies

$$
\frac{4}{9 \sqrt{3}} \leq \pi_{2}\left(\mathcal{K}_{4}^{-}\right) \leq \frac{3}{10}
$$

Proof: The lower bound follows from a simple construction. For the upper bound let $\mathcal{F}=((A, B), E)$ be a bipartite $\mathcal{K}_{4}^{-}$-free 3 -graph with $n$ vertices and $m$ edges. Let $|A|=\alpha n$, so $|B|=(1-\alpha) n$. Then

$$
\sum_{x y \in A \times B} d_{x y}=2 m, \quad \sum_{x y \in A^{(2)} \cup B^{(2)}} d_{x y}=m .
$$

From (1) and the fact that $q_{1} \geq 0$ we obtain

$$
m n \geq \sum_{x y \in A \times B} d_{x y}^{2}+\sum_{x y \in A^{(2)} \cup B^{(2)}} d_{x y}^{2}
$$

Convexity and (2) then imply that

$$
m \leq \frac{n^{3}}{\frac{4}{\alpha(1-\alpha)}+\frac{2}{\alpha^{2}+(1-\alpha)^{2}}} \leq \frac{n^{3}}{20} .
$$

The result then follows directly.

With some more work we can obtain an upper bound for $\pi_{3}\left(\mathcal{K}_{4}^{-}\right)$. (Again the lower bound follows from a simple construction.) 
Theorem 4 The 3-chromatic Turán density $\pi_{3}\left(\mathcal{K}_{4}^{-}\right)$satisfies

$$
\frac{5}{18} \leq \pi_{3}\left(\mathcal{K}_{4}^{-}\right)<\frac{58}{177}
$$

\section{References}

[1] D. de Caen, Extension of a theorem of Moon and Moser on complete subgraphs, Ars Combinatoria 16 (1983) 5-10.

[2] D. de Caen and Z. Füredi, The maximum size of 3-uniform hypergraphs not containing a Fano plane, Journal of Combinatorial Theory Series B, 78 (2000) 274-276.

[3] F. Chung and L. Lu, An upper bound for the Turán number $t_{3}(n, 4)$, Journal of Combinatorial Theory Series A, (1999) 87 381-389.

[4] P. Erdős and M. Simonovits, Supersaturated graphs and hypergraphs, Combinatorica 3 (1983) 181192.

[5] P. Erdôs and V.T. Sós, On Ramsey-Turán type theorems for hypergraphs, Combinatorica 2 (1982) 289-295.

[6] P. Frankl and Z. Füredi, An exact result for 3-graphs, Discrete Math. 50 (1984), no. 2-3, 323-328.

[7] P. Frankl and Z. Füredi, A new generalization of the Erdôs-Ko-Rado theorem, Combinatorica 3 (1983) 341-349.

[8] Z. Füredi, O. Pikhurko and M. Simonovits, The Turán density of the hypergraph $a b c, a d e, b d e, c d e$, Electronic Journal of Combinatorics, 10 (2003) 7pp

[9] D. Mubayi, On hypergraphs with every four points spanning at most two triples, Electronic Journal of Combinatorics 10 (2003), \#N10

[10] D. Mubayi and V. Rödl, On the Turán number of triple systems, Journal of Combinatorial Theory, Series A 100 (2002) 136-152.

[11] P. Turán, On an extremal problem in graph theory, Mat. Fiz. Lapok 48 (1941) 436-452 [in Hungarian].

[12] P. Turán, Turán memorial volume, Journal of Graph Theory 1 (2) (1977). 